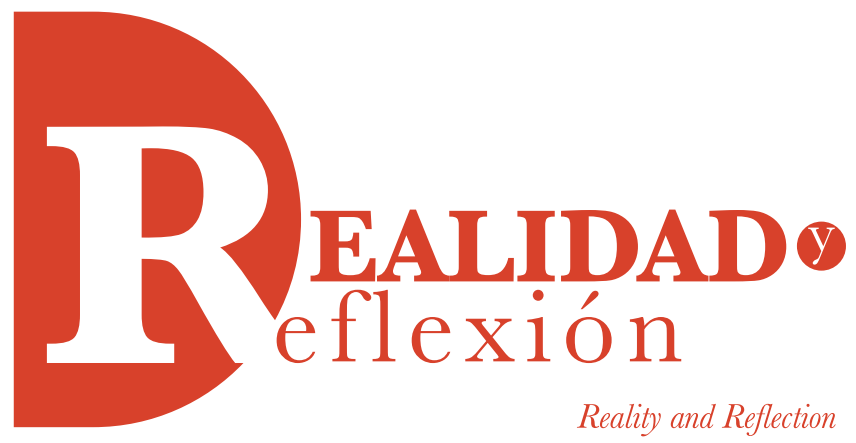

ISSN 1992-6510

e-ISSN 2520-92990

Año 17, No 45, San Salvador, El Salvador, Centroamérica. Revista Semestral Enero-Junio 2017

YEAR 17, N 45, SAN SALVADOR, EL SALVADOR, CENTRAL AMERICA. SEMESTRAL JOURNAL JANUARY-JUNE 2017

\title{
Medición de la cantidad de residuos plásticos pequeños en algunas playas de E1 Salvador
}

\author{
Mesaurement of plastic wastes in some beaches \\ of El Salvador
}

Dr. Enrique Barraza
Ph.D. en Ciencias Biológicas, opción Ecosistemas y Recursos Biológicos, Universidad de
Santiago de Compostela, España.
Master of Science, Zoología (Beca Fullbright), Universidad Texas A\&M, Estados Unidos.
Licenciado en Biología. Universidad de El Salvador, El Salvador.
Investigador asociado ICTI - UFG.

Fecha de recepción: 5 de enero de 2017

Fecha de aprobación: 28 de abril de 2017

\section{RESUMEN}

Durante los meses de abril y agosto de 2016, se recolectaron 24 muestras de arena intermareal y berma $(38,400 \mathrm{~cm} 2)$ en seis playas de El Salvador para medir el peso de fragmentos de plástico y otros residuos sólidos asociados. El sedimento se clasificó en tamaños superiores a 10, 4 y $2 \mathrm{~mm}$ en tamices separados. El material más abundante fueron restos vegetales que alcanzaron $376.6 \mathrm{~g}$, en cambio, se registraron $21.7 \mathrm{~g}$ de fragmentos plásticos. Debido a la cercanía con la vacación de Semana Santa de 2016, con el primer muestreo se detectó una tendencia de mayor acumulación de sólidos plásticos. Se detectó diferencia significativa al comparar los pesos de material vegetal y plástico entre estación seca y lluviosa. Por el contrario, los pesos de los diferentes desechos sólidos no presentaron diferencias significativas entre el área intermareal y berma.

Palabras clave: arena, berma, El Salvador, plásticos, playa.

ABSTRACT

I collected 24 intertidal and berm sand samples $(38,400 \mathrm{~cm} 2)$ on six beaches at El Salvador to measure weight of plastic fragments and other associated solid residues. Sediment was divided into larger sizes than 10, 4, and $2 \mathrm{~mm}$ through separated sieves. Most abundant material were plant debris that reached $376.6 \mathrm{~g}$, while, plastic fragments weighed $21.7 \mathrm{~g}$. A trend of higher plastic solids accumulation was associated to Holy Week 2016 proximity to first sampling season. Comparison of plant debris and plastics among the dry and rainy season showed significant differences. On the contrary, the weights of different wastes didn't present significant differences between intertidal area and berm.

Keywords: sand, berm, El Salvador, plastic fragments, beach. 


\section{Introducción}

La contaminación de los ecosistemas terrestres y acuáticos es una problemática global que está asociada a actividades humanas y procesos naturales. En general, cuando la literatura actual se refiere a alteraciones en los diferentes ecosistemas terrestres o marinos se utilizan dos términos que la lengua inglesa diferencia: contaminación y polución.

La primera ocurre cuando los aportes derivados de actividades humanas incrementan la concentración de una substancia en el agua, sedimentos, organismos, arriba de los niveles naturales de un área u organismos. En cambio, se denomina polución a la introducción antropogénica en forma directa o indirecta de substancias o formas de energía al ambiente natural que causa efectos perjudiciales como daños a la salud humana, restricción de actividades recreativas o productivas (Clark, 2001). La diferencia entre ambas definiciones es la afectación a la población humana, que se menciona en la segunda. Sin embargo, existen otras definiciones según la zona geográfica, donde los investigadores crecieron y desarrollaron. En América Latina, el vocablo más utilizado es contaminación.

Unos de los principales contaminantes globales derivados de los hidrocarburos son los plásticos que se convierten en desechos sólidos de origen antropogénico, los cuales se crearon para sustituir materiales que debido a su consumo se estaban volviendo escasos, por ejemplo la seda y el marfil. Los plásticos son materiales versátiles, de bajo peso, resistentes, transparentes; también, presentan bajo costo, aíslan humedad y oxígeno, lo que favorece su uso para empaques. Algunos de estos materiales incluyen polietileno $(\mathrm{PE})$, polipropileno (PP), poliestireno (PS), tereftalato de polietileno (PET), cloruro de polivinilo (PVC) y aditivos industriales (Cole et al, 2011).

Durante el último medio siglo, se ha incrementado el consumo de plásticos a nivel mundial, por lo que la acumulación de este tipo de residuo sólido es observable a nivel global. Al principio la problemática se consideró de índole estético y posteriormente se determinó su ocurrencia en tractos digestivos de aves playeras (van Franeker y Lavender-Law, 2015), así como como causa de morbilidad y mortandad de fauna, incluyendo la acuática como invertebrados y peces (Andrady, 2009; Sussarellu et al, 2016). La asociación PlasticsEurope (2016) determinó que la producción global de plásticos fue de 322 millones de toneladas en el año 2015. También, se estimó que la generación de desechos plásticos a nivel mundial en el año 2006 fue de 225 toneladas métricas por año (Barnes et al, 2009). Algunos estudios sobre este tipo de desechos en el océano indican que cada tipo de plástico tiene la capacidad de absorber o liberar contaminantes (Hirai et al, 2011; Zarfl et al, 2011).

El Salvador, como otras naciones del mundo, presenta un importante consumo de material plástico, cuyas partículas fragmentadas se acumulan en diferentes ecosistemas, incluyendo la franja litoral. En El Salvador, Christoph et al (2015) comprobaron la ocurrencia de microplásticos en agua superficial del océano Pacífico en el área natural protegida Complejo Los Cóbanos. Sin embargo, no existen estudios nacionales de cuantificación de estos fragmentos en la zona intermareal. Observaciones personales permiten confirmar la presencia de plásticos fragmentados y otros desechos sólidos 
flotantes y en el fondo del Golfo de Fonseca, Bahía de Jiquilisco (particularmente la zona de confluencia del río Grande de San Miguel), río Goascorán, río Pasaquina, río Lempa, entre otros.

La invasión del material plástico en el océano incluye su ocurrencia en el agua, sedimentos, la zona de playa que se ubica entre la línea de marea alta y baja, e incluso en el espacio de escasa pendiente entre la playa y duna o tierra continental que aún incluye arena de playa, denominada berma (McDermid y McMullen, 2004; Imhof et al, 2013; Fauziah et al, 2015).

\section{Material y método}

Las playas de donde se recolectó sedimento fueron: Los Cóbanos, Barra Ciega, E1 Pital (Mizata), Las Flores, Costa del Sol y El Espino, según se detalla en el Mapa n. ${ }^{\circ}$ 1. Se seleccionaron por su acceso libre mediante automóvil. Los muestreos del material litoral se realizaron el 25 y 29 de abril de 2016 (final de la época seca), así como el 29 y 31 de agosto del mismo año. (Ver Mapa n. 1).

Basado en el estudio de McDermid y McMullen (2004), la toma de muestras se realizó mediante el establecimiento de un área de 40 x $40 \mathrm{~cm}$ $\left(1,600 \mathrm{~cm}^{2}\right)$ tanto en la franja de marea alta, como en la zona supralitoral (adyacente a la anterior del lado terrestre), conocida como berma. Cada muestra de sedimento se removió con una pala hasta una profundidad de $5.5 \mathrm{~cm}$. El total de muestras (24) incluyó una superficie de $38,400 \mathrm{~cm}^{2}$. Posteriormente se determinó el peso con balanza digital apropiada AWS (H110). Este material se filtró en tamices de 10, 4 y $2 \mathrm{~mm}$. El material retenido en cada tamiz se clasificó en diferentes tipos de material: vegetal, arena, plástico, poliestireno, hule. Cada submuestra se lavó con agua para eliminar los restos de sedimentos y recolectar el material antes mencionado. Posteriormente se secaron por una hora a $65^{\circ} \mathrm{C}$.

Cada tipo de material sólido retenido se pesó en balanza analítica de laboratorio (OHAUS). Para reducir la interferencia de la humedad ambiental de las muestras de plásticos y otro material retenido, se mantuvieron en recipientes sellados con sobres de gel sílica comercial.

Las comparaciones estadísticas se realizaron mediante la prueba t-student, considerando el tamaño de los datos: 36 observaciones en estación seca y lluviosa, respectivamente.

\section{Resultados}

A continuación se detallan los resultados obtenidos en esta investigación. El cuadro n. ${ }^{\circ} 1$ presenta los pesos secos de la arena de playa recolectada en abril y agosto de 2016. (Ver Cuadro n. ${ }^{\circ}$ ).

El cuadro n. ${ }^{\circ} 2$ presenta los pesos secos del material sólido fragmentado (plástico, poliestireno y restos vegetales) retenido en el tamiz de $10 \mathrm{~mm}$. E1 material que alcanzó mayor ocurrencia fue el resto vegetal en comparación al plástico y poliestireno (durapax). La misma tendencia se muestra en el material tamizado de 4 y $2 \mathrm{~mm}$ (cuadros 3 y 4 , respectivamente). Los porcentajes de los residuos sólidos retenidos en relación con el peso total de la muestra de sedimento son bajos, particularmente poliestireno y plástico: la mayoría se presentó menor al 0.1\%. También, se observó que la fracción de fragmento que contenía mayor peso fue la mayor de $10 \mathrm{~mm}$ en los diferentes casos. 


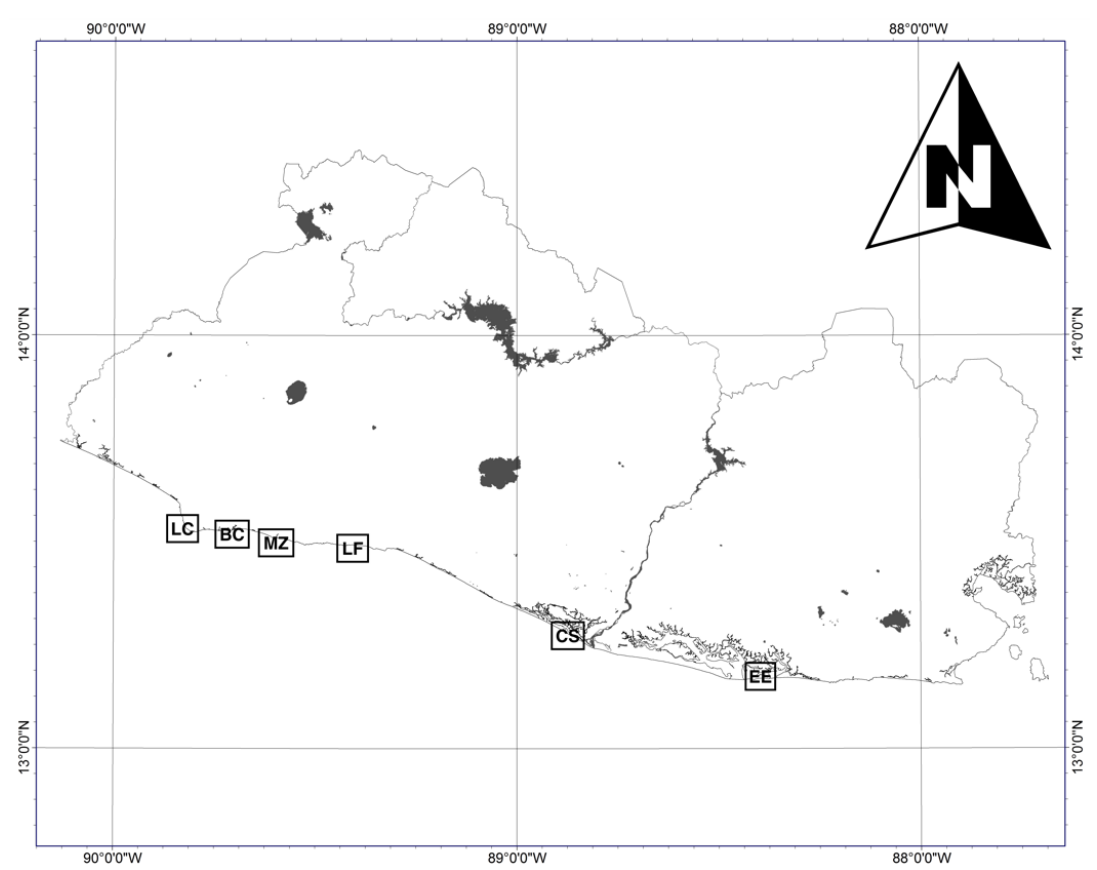

Mapa n. ${ }^{\circ}$ 1. Ubicación de las estaciones de muestreo. LC: Los Cóbanos, BC: Barra Ciega, MZ: Mizata, LF: Las Flores, CS: Costa del Sol, EE: El Espino. N: Norte.

\begin{tabular}{|c|c|c|}
\hline \multirow{2}{*}{ PLAYA } & \multicolumn{2}{|c|}{ PESO $(\mathrm{g})$} \\
\cline { 2 - 3 } & ABRIL 2016 & AGOSTO 2016 \\
\hline Los Cóbanos (BE) & 16515.7 & 14940.1 \\
\hline Los Cóbanos (LM) & 16827.9 & 15180.5 \\
\hline Barra Ciega ((BE) & 11329.6 & 11120.6 \\
\hline Barra Ciega (LM) & 10637.4 & 13840.3 \\
\hline Mizata (BE) & 12952.9 & 11400.5 \\
\hline Mizata (LM) & 14093.9 & 11860.3 \\
\hline Las Flores (BE) & 13247.8 & 13080.4 \\
\hline Las Flores (LM) & 12352.5 & 14050.8 \\
\hline Costa del Sol (BE) & 16580.0 & 11500.3 \\
\hline Costa del Sol (LM) & 12405.4 & 11327.5 \\
\hline E1 Espino (BE) & 16321.9 & 14997.9 \\
\hline E1 Espino (LM) & 15007.8 & 14066.7 \\
\hline Total & 168272.8 & 157365.9 \\
\hline
\end{tabular}

\section{Cuadro n. ${ }^{\circ} 1$}

Peso del sedimento de las playas sin incluir los desechos sólidos. BE: Berma, LM: línea de marea alta 


\begin{tabular}{|c|c|c|c|c|c|c|c|c|c|c|c|c|c|}
\hline MAT & Los & óbanos & Barra & Ciega & $\mathrm{Mi}$ & & Las & lores & Costa & de Sol & El E & spino & \\
\hline$\overline{A B R}$ & $\mathrm{BE}$ & $\overline{L M}$ & $\overline{B E}$ & LM & $\mathrm{BE}$ & $\overline{L M}$ & $\overline{B E}$ & \begin{tabular}{|l} 
LM \\
\end{tabular} & $\overline{B E}$ & LM & $\overline{B E}$ & LM & $\Sigma$ \\
\hline PLA & 0.0 & 0.0 & 0.0 & 0.0 & 0.0 & 0.1 & 5.9 & 0.0 & 0.0 & 0.0 & 5.2 & 1.7 & 12.9 \\
\hline$\%$ & 0.0 & 0.0 & 0.0 & 0.0 & 0.0 & $<0.1$ & $<0.1$ & 0.0 & 0.0 & 0.0 & $<0.1$ & $<0.1$ & $<0.1$ \\
\hline POL & 0.0 & 0.0 & 0.0 & 0.0 & 0.0 & 0.1 & 0.2 & 0.0 & 0.0 & 0.0 & 0.1 & 0.1 & 0.5 \\
\hline$\%$ & 0.0 & 0.0 & 0.0 & 0.0 & 0.0 & $<0.1$ & $<0.1$ & 0.0 & 0.0 & 0.0 & $<0.1$ & $<0.1$ & $<0.1$ \\
\hline RVG & 3.0 & 4.5 & 2.4 & 0.0 & 0.0 & 3.8 & 27.9 & 0.0 & 0.0 & 0.0 & 58.0 & 100.0 & 199.6 \\
\hline & $<0.1$ & $<0.1$ & $<0.1$ & 0.0 & 0.0 & $<0.1$ & $<0.1$ & 0.0 & 0.0 & 0.0 & 0.4 & 0.7 & 0.1 \\
\hline $\mathrm{AGO}$ & & & & & & & & & & & & & \\
\hline PLA & 0.0 & 0.3 & 0.0 & 0.0 & 0.0 & 0.3 & 0.0 & 0.0 & 0.7 & 0.0 & 2.7 & 0.0 & 4.0 \\
\hline$\%$ & 0.0 & $<0.1$ & 0.0 & 0.0 & 0.0 & $<0.1$ & $<0.1$ & 0.0 & $<0.1$ & 0.0 & $<0.1$ & 0.0 & $<0.1$ \\
\hline POL & 0.0 & 0.0 & 0.0 & 0.0 & 0.0 & 0.0 & 0.4 & 2.3 & 0.0 & 0.0 & 0.4 & 0.0 & 3.1 \\
\hline$\%$ & 0.0 & 0.0 & 0.0 & 0.0 & 0.0 & 0.0 & $<0.1$ & $<0.1$ & 0.0 & 0.0 & $<0.1$ & 0.0 & $<0.1$ \\
\hline RVG & 0.1 & 11.1 & 0.0 & 1.5 & 0.4 & 0.0 & 15.6 & 2.5 & 0.0 & 0.3 & 36.8 & 0.0 & 68.3 \\
\hline$\%$ & $<0.1$ & $<0.1$ & $<0.1$ & $<0.1$ & $<0.1$ & 0.0 & $<0.1$ & $<0.1$ & 0.0 & $<0.1$ & 0.2 & 0.0 & $<0.1$ \\
\hline
\end{tabular}

\section{Cuadro n. ${ }^{\circ} 2$}

Peso seco (g) del material sólido retenido en el tamizado de $10 \mathrm{~mm}$. PLA: plásticos, POL: poliestireno, RVG: resto vegetal. ABR: Abril, AGO: agosto.

\begin{tabular}{|c|c|c|c|c|c|c|c|c|c|c|c|c|c|}
\hline MAT & Los & óbanos & Barr & Ciega & $\mathrm{Mi}$ & & Las & lores & Costa & de Sol & El E & pino & \\
\hline $\mathrm{ABR}$ & $\mathrm{BE}$ & LM & $\mathrm{BE}$ & LM & $\mathrm{BE}$ & LM & $\mathrm{BE}$ & LM & $\mathrm{BE}$ & LM & $\mathrm{BE}$ & LM & $\Sigma$ \\
\hline PLA & 0.0 & 0.0 & 0.0 & 0.0 & 0.0 & 0.0 & 0.6 & 0.0 & 0.0 & 0.0 & $\overline{1.4}$ & 0.0 & 2.0 \\
\hline$\%$ & 0.0 & 0.0 & 0.0 & 0.0 & 0.0 & 0.0 & $<0.1$ & 0.0 & 0.0 & 0.0 & $<0.1$ & $<0.1$ & $<0.1$ \\
\hline POL & 0.0 & 0.0 & 0.0 & 0.0 & 0.0 & 0.0 & 0.1 & 0.0 & 0.0 & 0.0 & 0.5 & 0.1 & 0.7 \\
\hline$\%$ & 0.0 & 0.0 & 0.0 & 0.0 & 0.0 & 0.0 & 0.1 & 0.0 & 0.0 & 0.0 & $<0.1$ & $<0.1$ & $<0.1$ \\
\hline RVG & 0.2 & 0.8 & 1.1 & 0.0 & 0.2 & 0.0 & 14.6 & 0.0 & 0.0 & 0.0 & 59.0 & 0.0 & 75.9 \\
\hline$\%$ & $<0.1$ & $<0.1$ & $<0.1$ & 0.0 & $<0.1$ & 0.0 & 0.1 & 0.0 & 0.0 & 0.0 & 0.4 & 0.0 & $<0.1$ \\
\hline $\mathrm{AGO}$ & & & & & & & & & & & & & \\
\hline PLA & 0.4 & 0.0 & 0.0 & 0.0 & 0.2 & 0.0 & 0.1 & 0.0 & 0.0 & 0.0 & 0.7 & 0.0 & 1.4 \\
\hline$\%$ & $<0.1$ & 0.0 & 0.0 & 0.0 & $<0.1$ & 0.0 & $<0.1$ & 0.0 & 0.0 & 0.0 & $<0.1$ & 0.0 & $<0.1$ \\
\hline POL & 0.0 & 0.0 & 0.0 & 0.0 & 0.0 & 0.0 & 0.3 & 0.0 & 0.0 & 0.0 & 0.2 & 0.0 & 0.5 \\
\hline$\%$ & 0.0 & 0.0 & 0.0 & 0.0 & 0.0 & 0.0 & $<0.1$ & 0.0 & 0.0 & 0.0 & $<0.1$ & 0.0 & $<0.1$ \\
\hline RVG & 0.2 & 2.3 & 0.2 & 0.3 & 0.0 & 0.1 & 8.3 & 3.9 & 0.0 & 3.0 & 15.4 & 0.0 & 33.7 \\
\hline$\%$ & $<0.1$ & $<0.1$ & $<0.1$ & $<0.1$ & 0.0 & $<0.1$ & $<0.1$ & $<0.1$ & 0.0 & 0.0 & $<0.1$ & 0.0 & $<0.1$ \\
\hline
\end{tabular}

\section{Cuadro n. ${ }^{\circ} 3$}

Peso seco (g) del material sólido retenido en el tamizado de $4 \mathrm{~mm}$. PLA: plásticos, POL: poliestireno, RVG: restos vegetales. 


\begin{tabular}{|c|c|c|c|c|c|c|c|c|c|c|c|c|c|}
\hline \multirow{2}{*}{$\frac{\text { MAT }}{\text { ABR }}$} & \multicolumn{2}{|c|}{ Los Cóbanos } & \multicolumn{2}{|c|}{ Barra Ciega } & \multicolumn{2}{|c|}{ Mizata } & \multicolumn{2}{|c|}{ Las Flores } & \multicolumn{2}{|c|}{ Costa de Sol } & \multicolumn{2}{|c|}{ E1 Espino } & \multirow[b]{2}{*}{$\Sigma$} \\
\hline & $\mathrm{BE}$ & LM & $\overline{B E}$ & LM & $\mathrm{BE}$ & LM & $\overline{\mathrm{BE}}$ & LM & $\mathrm{BE}$ & $\overline{L M}$ & $\overline{B E}$ & LM & \\
\hline PLA & 0.1 & 0.0 & 0.0 & 0.0 & 0.0 & 0.0 & 0.0 & 0.0 & 0.0 & 0.0 & 0.5 & 0.0 & 0.6 \\
\hline$\%$ & $<0.1$ & 0.0 & 0.0 & 0.0 & 0.0 & 0.0 & 0.0 & 0.0 & 0.0 & 0.0 & $<0.1$ & 0.0 & $<0.1$ \\
\hline $\mathrm{POL}$ & 0.0 & 0.0 & 0.0 & 0.0 & 0.0 & 0.0 & 0.0 & 0.0 & 0.0 & 0.0 & 0.4 & 0.0 & 0.5 \\
\hline$\%$ & 0.0 & 0.0 & 0.0 & 0.0 & 0.0 & 0.0 & 0.0 & 0.0 & 0.0 & 0.0 & $<0.1$ & 0.0 & $<0.1$ \\
\hline RVG & 1.4 & 0.1 & 1.1 & 0.1 & 0.2 & 0.0 & 0.0 & 0.0 & 0.0 & 0.0 & 64.2 & 0.0 & 67.3 \\
\hline$\%$ & $<0.1$ & $<0.1$ & $<0.1$ & $<0.1$ & $<0.1$ & 0.0 & 0.0 & 0.0 & 0.0 & $\overline{0.0}$ & $\overline{0.4}$ & 0.0 & $<0.1$ \\
\hline \multicolumn{14}{|l|}{$\mathrm{AGO}$} \\
\hline$\overline{\text { PLA }}$ & 0.1 & 0.3 & 0.0 & 0.0 & 0.0 & 0.0 & 0.1 & 0.0 & 0.0 & $\overline{0.0}$ & 0.3 & 0.0 & 0.8 \\
\hline$\%$ & $<0.1$ & $<0.1$ & 0.0 & 0.0 & 0.0 & 0.0 & $<0.1$ & 0.0 & 0.0 & 0.0 & $<0.1$ & 0.0 & $<0.1$ \\
\hline$\overline{\mathrm{POL}}$ & 0.0 & 0.0 & 0.0 & 0.0 & 0.0 & 0.0 & 0.1 & 0.0 & 0.0 & 0.0 & 0.3 & 0.0 & 0.4 \\
\hline$\%$ & 0.0 & 0.0 & 0.0 & 0.0 & 0.0 & 0.0 & $<0.1$ & 0.0 & 0.0 & 0.0 & $<0.1$ & 0.0 & $<0.1$ \\
\hline RVG & 0.0 & 2.1 & 0.0 & 0.2 & 0.0 & 0.0 & 13.1 & 0.0 & 0.0 & 0.0 & 16.0 & 0.0 & 31.4 \\
\hline$\%$ & 0.0 & $<0.1$ & 0.0 & $<0.1$ & 0.0 & 0.0 & $<0.1$ & 0.0 & 0.0 & 0.0 & $<0.1$ & 0.0 & $<0.1$ \\
\hline
\end{tabular}

\section{Cuadro n. ${ }^{\circ} 4$}

Peso seco $(g)$ del material sólido retenido en el tamizado de $2 \mathrm{~mm}$. PLA: plásticos, POL: poliestireno, RVG: restos vegetales

\section{Discusión}

Los resultados antes mencionados son opuestos a los determinados por Mcdermid y McMullen (2004) en playas de las islas de Hawaii, quienes hallaron mayor cantidad de plásticos $(532.4 \mathrm{~g}$ en $81,862 \mathrm{~cm}^{2}$ de superficie) en playas y bermas, en comparación a $21.7 \mathrm{~g}$ de residuos plásticos y $376.6 \mathrm{~g}$ de material vegetal en $38,400 \mathrm{~cm} 2$. También, el peso de plásticos detectado por Fauziah et al (2015) en seis playas de Malasia reflejó valores más altos $\left(265.3 \mathrm{~g} / \mathrm{m}^{2}\right)$ en comparación a este estudio $\left(5.7 \mathrm{~g} / \mathrm{m}^{2}\right)$.

La posible explicación podría asociarse, en parte, a que en las playas incluidas en este estudio se recolectó una menor cantidad de sedimento en comparación con los estudios mencionados, y además se utiliza material vegetal duro como combustible para la cocción de alimentos y en la construcción de infraestructura temporal. Gran parte de las muestras vegetales analizadas presentaron evidencia de combustión reciente.

La comparación entre el total de residuos sólidos recolectados en la línea de marea alta en comparación con la berma, determinó que no existen diferencias significativas entre los tipos de material y su tamaño (Cuadro n. ${ }^{\circ}$ 5). Lo cual refleja la influencia de las actividades humanas en la zona de berma y posiblemente la dispersión mecánica del mismo material que realizan las mareas altas, como Fauziah et al (2015) detectaron. (Ver Cuadro n. ${ }^{\circ}$ 5)

También se determinaron diferencias significativas ( $p>0.05$ ) en los pesos secos de plástico y material vegetal entre las épocas seca y lluviosa, no así para 


\begin{tabular}{|l|r|r|r|}
\hline \multirow{2}{*}{ MATERIAL SÓLIDO TAMIZADO } & \multicolumn{3}{|c|}{ TAMAÑ DEL TAMIZADO } \\
\cline { 2 - 4 } & BE-LM 10 mm & BE-LM 4 mm & BE-LM 2 mm \\
\hline Plástico & $\mathrm{ns}$ & $\mathrm{ns}$ & $\mathrm{ns}$ \\
\hline Poliestireno & $\mathrm{ns}$ & $\mathrm{ns}$ & $\mathrm{ns}$ \\
\hline Material vegetal & $\mathrm{ns}$ & $\mathrm{ns}$ & $\mathrm{ns}$ \\
\hline
\end{tabular}

\section{Cuadro n. ${ }^{\circ} 5$}

Comparación de los pesos secos $(g)$ de plástico, poliuretano y restos vegetales entre las bermas y líneas de marea alta, según los tamaños de tamizado. BE: Berma, LM: línea de marea alta. Prueba T-student, dos colas $(p<0.05)$.

\begin{tabular}{|l|r|r|}
\hline MATERIAL RETENIDO EN 12 MUESTRAS DE SEDIMENTO & \multicolumn{2}{|c|}{ PESO (g) } \\
\hline & ABRIL & AGOSTO \\
\hline Plástico* $^{*}$ & 15.5 & 6.2 \\
\hline Poliestireno (ns) & 1.4 & 4.0 \\
\hline Material vegetal* & 343.7 & 133.4 \\
\hline Total & 360.6 & 143.6 \\
\hline
\end{tabular}

\section{Cuadro n. ${ }^{\circ} 6$}

Peso seco total $(g)$ de material sólido retenido en las 24 muestras $(38,400 \mathrm{~cm} 2)$. *diferencia significativa ( $p<0.05)$, T-Student dos colas, ns: diferencia no significativa ( $p>0.05)$.

el poliestireno (Cuadro n. ${ }^{\circ}$ 6). Lo cual indica que ambos tipos de fragmentos presentan mayor deposición durante la primera época, posiblemente asociado a la vacación de Semana Santa (20-27 marzo 2016) que atrajo un elevado número de turistas en el litoral salvadoreño, así como a la ausencia de lluvias y mareas extraordinarias, que no dispersaron el material.

Las figuras números 1, 2 y 3 reflejan los niveles acumulados de material fragmentado de plástico, poliestireno y resto vegetal, respectivamente. Es evidente que las playas "Las Flores"y "E1 Espino" presentan mayores niveles de esos residuos. En ambos casos las estaciones de recolecta de arena se encontraban cerca de infraestructura temporal o permanente de infraestructura turística, donde no existe un manejo apropiado de los residuos sólidos generados.

Un hallazgo inesperado en la muestra de época lluviosa, de línea de marea más alta de Costa del Sol, fue la presencia de un espécimen vivo de la especie Excirolana brasiliensis Richardson, 1912 (Isopoda: Cirolanidae), después de 21 días de mantener la muestra de arena dentro de una cubeta plástica bajo sombra, en la ciudad de San Salvador. $\mathrm{Su}$ presencia en playas arenosas adyacentes al océano Pacífico en Punta San Juan, Usulután, se confirmó por Barraza-Sandoval (2010). 


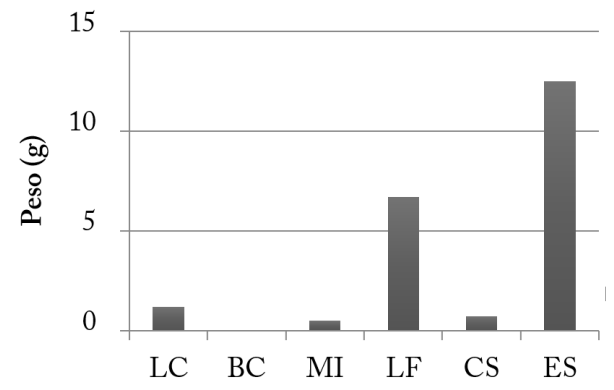

Figura $n .^{\circ}$ 1. Peso total acumulado de fragmentos plásticos en seis playas de El Salvador.

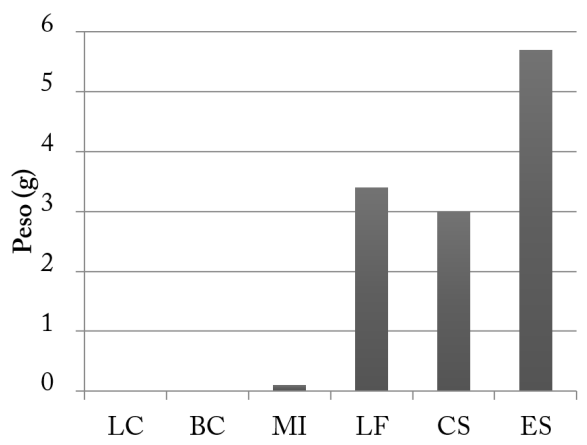

Figura $n .^{\circ}$ 2. Peso total acumulado de fragmentos de poliestireno en seis playas de E1 Salvador.

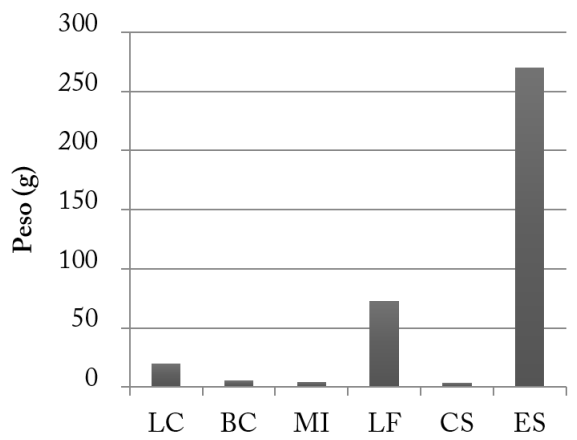

Figura $n .^{\circ} 3$. Peso total acumulado de restos vegetales en seis playas de E1 Salvador.
La tendencia general detectada con estos resultados es que las playas de mayor afluencia humana, como las playas "Las Flores" y "El Espino", en este caso turismo de playa, son las que presentaron mayor cantidad de plásticos como Corcoran et al, 2008 y Jambeck et al, 2015 lo han propuesto. Otro hallazgo importante es que la cantidad en peso de material sólido (gramos) detectado en un total de $38,400 \mathrm{~cm}^{2}$ de sedimento arenoso (24 muestras, casi $4 \mathrm{~m}^{2}$ ), presenta un patrón definido de abundancia que se presenta de la siguiente forma descendente: material vegetal > plástico > poliestireno. Además se pudo determinar que tanto la cantidad en peso de los tres tipos de residuos es similar en la línea de marea más alta y la berma, siendo un poco diferente al comparar la época seca y lluviosa, ya que fueron diferentes los pesos secos del plástico y material vegetal, y se mantuvieron similares los del poliestireno, reflejando alguna interacción del viento, lluvias, corrientes, mareas y actividades turísticas. Esta última práctica se asocia a la quema de material leñoso que se utiliza para cocción de alimentos, particularmente en las playas mencionadas anteriormente.

Se requieren monitoreos que incluyan otras épocas del año para determinar lo que ocurre en la época seca.

\section{Conclusiones}

Se detectaron diferentes residuos sólidos principales durante el período de estudio que se ordenan en forma decreciente según su abundancia por peso: material vegetal $(98.1 \mathrm{~g} /$ $\left.\mathrm{m}^{2}\right)$, plástico $\left(5.7 \mathrm{~g} / \mathrm{m}^{2}\right)$, poliestireno $\left(0.6 \mathrm{~g} / \mathrm{m}^{2}\right)$. 
Las playas que presentaron mayor cantidad de residuos plásticos fueron "Las Flores" y "El Espino", donde la actividad turística en intensa.

No existe diferencia significativa entre la abundancia en peso del material mencionado entre la zona intermareal y la berma de playa, reflejando el área de berma y supralitoral, como la zona donde se generan desechos sólidos que afectan en gran parte a las playas en estudio.

Sí existieron diferencias significativas al comparar el peso registrado del material vegetal y plástico entre época seca y lluviosa, donde factores meteorológicos, aparte de los antropogénicos, pueden combinarse para influir en la distribución de este tipo de residuos sólidos.

\section{Referencias}

Andray, A.I. (2011). Microplastics in the marine environment. Marine Pollution Bulletin 62: 1596-1605.

Barnes, D.K.A., Galcani, F. Thompson, R.C. y Barlaz, M. (2009). Accumulation and fragmentation of plastic debris in global environments. Phylosophical Transactions of the Royal Society 364: 1985-1998.

Barraza-Sandoval, J.E. (2010). Biodiversidad de Bahía de Jiquilisco. En: C.G. Rivera \& T. C. Cuéllar. El Ecosistema de Manglar de la Babia de Jiquilisco. Universidad de E1 Salvador. 13-32, 231

Clark, R.B. 2001. Marine Pollution, 5th Ed, p.237. Oxford University Press.
Christoph, R., Hernández, A., Muñoz, R. y Picardo, O. (2015). Microplásticos en El Salvador. En Nanotecnia: Febrero. Instituto de Ciencia, Tecnología e Innovación. Universidad Francisco Gavidia. 6 pp. <disponible en: http://nanotecnialab.ufg.edu.sv/uploads/ newsletters/2016-02-01.pdf>

Cole, M., Lindeque. P, Halsband, C., Galloway, T.S. (2011). Microplastics as contaminants in the marine environment: A Review. Marine Pollution Bulletin 62: 2588-2597.

Corcoran, P.L, Orcoran, P. L. ; Biesinger, M.C. Grifi, M. (2009). Plastics and beaches: A degrading relationships. Marine Pollution Bulletin 58: 80-84.

Fauzia, S.H., Liyana, I.A., Agamuthu, P. (2015). Plastic debris in the coastal environment: The invincible threat? Abundance of buried plastic debris on Mayaysian beaches.

Jambeck, J.R., Geyer, R., Wilcox. C., Ambeck, J.R., Siegler, T.R., Perryman, M., Adrady, A., Narayan, R., Law, K.L. (2015). Plastic waste inputs from land into the ocean. Science 347: 768-771.

Hirai, H., Takada, H., Ogata, Y., Yamashita, R., Mizukawa, K., Saha, M., Kwan, C., Moore, C., Grat, H., Laursen, D., Zettler, E.R., Farrington, J.W., Reddy, C.M., Peacock, E.E., Ward, M.W. (2011). Organic micropollutants in marine plastics debris from the open ocean and remote and urban areas. Marine Pollution Bulletin 62: 1863-1692. 
Imhof, H.K., Ivleva, N.P., Schimd, J., Niessner, R., Laforsch, C. (2013). Contamination of beach sediments of a subalpine lake with microplastic particles. Current Biology: 23: 867-868.

McDermid, K.J., McMullen, T.L. (2004). Quatitative análisis of small-plastic debris on beaches in the Hawaiian archipelago. Marine Pollution Bulletin 48:790-794.

Plasticseurope. (2016). Plastics - The Facts 2016. An Analysis of European plastics, production, demand and waste data. diponible en: http://www.plasticseurope.org/documents/ document/20161014113313-plastics_the_ facts_2016_final_version.pdf
Sussarellu, R., Suqueto M., Thomas Y., Lambert C., Fabious, C., Pernet, M.E.J., Le Goic, N., Quillien, V., Mingant, C., Epelboin, Y., Corporeau, C., Guyomarch, J., Robbens, H., Paul-Pont, I., Soudant, P., Huvet, A. (2016). Oyster reproduction is affected by exposure to polystirene microplastics. Proceeding of the National Academy of Sciences 113: 2430-2435.

Van Franeker, J.A., Lavender-Law, K. (2015). Seabirds, gyres and global trends in plastic pollution. Environmental Pollution 203: 89-96.

Zarfl, C., Fleet, D., Fries, E., Matthies, M. (2011). Microplastics in oceans. Marine Pollution Bulletin 62: 1589-1591. 